\title{
Tailoring the magnetic anisotropy of CoFeB/ MgO stacks onto W with a Ta buffer layer
}

Cite as: Appl. Phys. Lett. 106, 262401 (2015); https://doi.org/10.1063/1.4923272

Submitted: 30 April 2015 . Accepted: 19 June 2015 . Published Online: 29 June 2015

Andreas Kaidatzis, Cristina Bran, Vasilios Psycharis, Manuel Vázquez, José Miguel García-Martín, and Dimitrios Niarchos
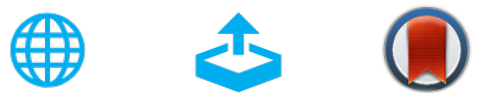

View Online

Export Citation

\section{ARTICLES YOU MAY BE INTERESTED IN}

Large enhanced perpendicular magnetic anisotropy in CoFeB/MgO system with the typical Ta buffer replaced by an Hf layer

AIP Advances 2, 032151 (2012); https://doi.org/10.1063/1.4748337

Spin transfer torque devices utilizing the giant spin Hall effect of tungsten

Applied Physics Letters 101, 122404 (2012); https://doi.org/10.1063/1.4753947

Perpendicular-anisotropy CoFeB-MgO magnetic tunnel junctions with a MgO/CoFeB/Ta/ $\mathrm{CoFeB} / \mathrm{MgO}$ recording structure

Applied Physics Letters 101, 022414 (2012); https://doi.org/10.1063/1.4736727

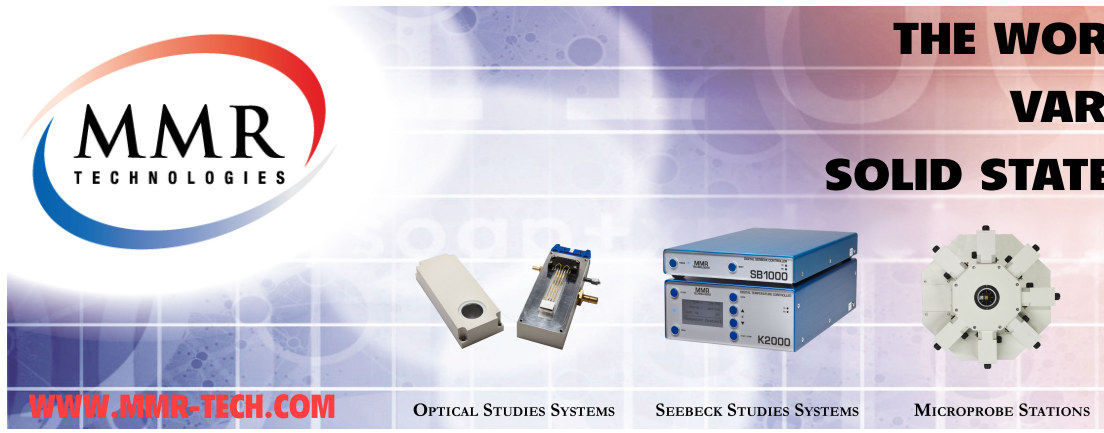
VARIABLE TEMPERATURE

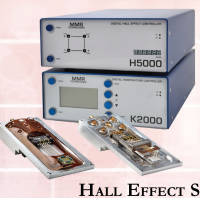

Hall Effect Study Systems and Magnet 


\title{
Tailoring the magnetic anisotropy of $\mathrm{CoFeB/MgO}$ stacks onto W with a Ta buffer layer
}

\author{
Andreas Kaidatzis, ${ }^{1, a)}$ Cristina Bran, ${ }^{2}$ Vasilios Psycharis, ${ }^{1}$ Manuel Vázquez, ${ }^{2}$ \\ José Miguel García-Martín, ${ }^{3}$ and Dimitrios Niarchos ${ }^{1}$ \\ ${ }^{1}$ Institute of Nanoscience and Nanotechnology, NCSR Demokritos, Aghia Paraskevi, Attikis, Athens 15310, \\ Greece \\ ${ }^{2}$ ICMM-Instituto de Ciencia de Materiales (CNM-CSIC), Sor Juana Inés de la Cruz 3, Cantoblanco, \\ E-28049 Madrid, Spain \\ ${ }^{3}$ IMM-Instituto de Microelectrónica de Madrid (CNM-CSIC), Isaac Newton 8, PTM, E-28760 Tres Cantos, \\ Madrid, Spain
}

(Received 30 April 2015; accepted 19 June 2015; published online 29 June 2015)

\begin{abstract}
The emergence of perpendicular magnetic anisotropy (PMA) in $\mathrm{CoFeB} / \mathrm{MgO}$ stacks deposited on $\mathrm{W}$ using a Ta buffer layer is studied as a function of Ta and CoFeB layer thickness and annealing temperature. It is shown that very thin Ta "dusting" layers (thickness between 0.3 and $1 \mathrm{~nm}$ ) enhance PMA of CoFeB layers grown on top of W. We find that Ta thickness is a crucial factor affecting magnetic anisotropy and it needs to be scaled proportionally to CoFeB thickness for obtaining PMA. Stacks without Ta have in-plane anisotropy, verifying the "PMA-enhancing" role of Ta. The maximum effective PMA energy $\left(3.6 \times 10^{6} \mathrm{erg} / \mathrm{cm}^{3}\right)$ is obtained for a stack with $1.4 \mathrm{~nm}$ of $\mathrm{CoFeB}$ and $1 \mathrm{~nm}$ of Ta and after annealing at $350^{\circ} \mathrm{C}$. Besides, PMA can be obtained even at the as-deposited state for certain thicknesses. This $\mathrm{W}$-based $\mathrm{CoFeB} / \mathrm{MgO}$ system could enable the development of low power consumption, high density, and non-volatile magnetic memories. (C) 2015 AIP Publishing LLC. [http://dx.doi.org/10.1063/1.4923272]
\end{abstract}

Perpendicular Magnetic Anisotropy (PMA) materials are currently under intense study because of their high potential for use in spintronics applications like Magnetic Random Access Memories (MRAMs), ${ }^{1}$ spin-transistors, ${ }^{2}$ and spinlogic devices. ${ }^{3}$ In particular, Magnetic Tunnel Junctions (MTJs), a stack of two ferromagnetic layers separated by an oxide, are key components of future spintronics technologies. One of the most promising MTJ materials systems is composed of $\mathrm{CoFeB} / \mathrm{MgO} / \mathrm{CoFeB}$ stacks, with PMA arising from the strong $\mathrm{CoFeB} / \mathrm{MgO}$ interface anisotropy. ${ }^{4}$ These MTJs show high tunnel magnetoresistance and a low threshold current for spin-transfer torque magnetization switching properties of particular importance for MRAM applications. What is more, PMA is a necessary requirement for ensuring high data stability and adequate scalability of the device.

Recently, Spin-Orbit Torque MRAM (SOT-MRAM) has been proposed as a novel memory concept ${ }^{5}$ promising a fast access, energy-efficient, scalable, high density, and nonvolatile memory technology. A typical SOT-MRAM stack is composed of a MTJ with PMA, grown on top of a high spinorbit coupling heavy-metal, which acts as the read/write current line. Although initial SOT-MRAM studies were focused on the $\mathrm{Pt} / \mathrm{Co} / \mathrm{AlO}_{x}$ system, ${ }^{6}$ there has been a shift towards $\mathrm{Ta} / \mathrm{CoFeB} / \mathrm{MgO}$ structures, due to the higher spin-orbit coupling of $\mathrm{Ta}$, the readily obtainable PMA of $\mathrm{CoFeB}$ after annealing and the more effective spin-dependent tunneling through the monocrystalline $\mathrm{MgO}$ barrier. ${ }^{4}$

Recent SOT-MRAM studies have been focused on $\mathrm{W}$, for use as current line metal, due to its reported giant spinorbit interaction $^{7}$ that allows the fabrication of even lower read/write current SOT-MRAM cells, enabling the industrial

a)Electronic mail: a.kaidatzis@inn.demokritos.gr application of SOT-MRAM technology. Thus, obtaining W/ $\mathrm{CoFeB} / \mathrm{MgO}$ stacks with good PMA is a hot topic in this field. For instance, Hao and $\mathrm{Xiao}^{8}$ perform annealing at $280{ }^{\circ} \mathrm{C}$ under a perpendicular magnetic field of $0.45 \mathrm{~T}$, i.e., with high energy consumption, for obtaining PMA. On the other hand, Torrejon and co-workers ${ }^{9}$ anneal their stacks at $300{ }^{\circ} \mathrm{C}$ without magnetic field, obtaining PMA when the $\mathrm{W}$ layers have thickness of $4 \mathrm{~nm}$ or less, but with a reduced $\mathrm{CoFeB}$ magnetization, while in-plane anisotropy (IPA) is obtained for $6 \mathrm{~nm}$ $\mathrm{W}$ thickness. Finally, Pai and collaborators ${ }^{10}$ have shown that strong PMA may be obtained only by inserting a thin Hf layer between the $\mathrm{W}$ and $\mathrm{CoFeB}$ layers.

In a similar context, we study W-based $\mathrm{CoFeB} / \mathrm{MgO}$ half-MTJ stacks (only the "free" MTJ layer is present), with a configuration in-between the ones mentioned above: we use a $6 \mathrm{~nm}$ thick $\mathrm{W}$ underlayer, which maintains a high CoFeB magnetization, but with an additional Ta "dusting" layer inserted between $\mathrm{W}$ and $\mathrm{CoFeB}$, whose thickness is systematically varied. It is shown that for certain parameters, PMA can be obtained even without annealing. The CoFeB/ $\mathrm{MgO}$ stack magnetic anisotropy is studied as a function of Ta layer thickness and annealing temperature, and it is shown that the Ta layer thickness needs to be proportionally scaled with the $\mathrm{CoFeB}$ thickness for effectively enhancing PMA. The maximum effective PMA energy $\left(3.6 \times 10^{6} \mathrm{erg} / \mathrm{cm}^{3}\right)$ is obtained for the sample with $1.4 \mathrm{~nm}$ of $\mathrm{CoFeB}$ and $1 \mathrm{~nm}$ of Ta, after annealing at $350{ }^{\circ} \mathrm{C}$ for an hour.

The samples studied were $\mathrm{W}(6 \mathrm{~nm}) / \mathrm{Ta}\left(t_{T a} \mathrm{~nm}\right) / \mathrm{Co}_{20} \mathrm{Fe}_{60}$ $\mathrm{B}_{20}\left(t_{C O F e B} \mathrm{~nm}\right) / \mathrm{MgO}(2 \mathrm{~nm}) / \mathrm{AlOx}(2 \mathrm{~nm})$ multilayers, where $t_{T a}$ $=0,0.3,0.6$, and $1 \mathrm{~nm}$ and $t_{C o F e B}=0.9,1.2,1.4$, and $1.8 \mathrm{~nm}$. CoFeB films with 20 at. \% B are deposited to produce an amorphous as-deposited structure, ${ }^{11}$ while $\mathrm{Co}_{20} \mathrm{Fe}_{60} \mathrm{~B}_{20}$ is reported ${ }^{12}$ to have stronger $\mathrm{CoFeB} / \mathrm{MgO}$ interface anisotropy than 
$\mathrm{Co}_{60} \mathrm{Fe}_{20} \mathrm{~B}_{20}$. All the stacks were covered by $\mathrm{AlO}_{x}$ for preventing oxygen diffusion through $\mathrm{MgO}$ and into $\mathrm{CoFeB}$.

Ultra-high vacuum (base pressure $1.5 \times 10^{-9}$ Torr) magnetron sputtering was employed for deposition on monocrystalline $\mathrm{Si}\langle 100\rangle$ substrates covered with an amorphous $500 \mathrm{~nm}$ thick thermal $\mathrm{SiO}_{2}$. Direct current (radio frequency) power supply was used for metal (oxide) deposition, at $1.5 \mathrm{~W} / \mathrm{cm}^{2}\left(10 \mathrm{~W} / \mathrm{cm}^{2}\right)$ power density and $3 \mathrm{mTorr}$ Ar working pressure, leading to $0.018 \mathrm{~nm} / \mathrm{s}(0.010 \mathrm{~nm} / \mathrm{s})$ deposition rate. The substrates were rotating during deposition for avoiding the emergence of any in-plane magnetic anisotropy axes. After deposition, the samples were annealed for $1 \mathrm{~h}$ at $250{ }^{\circ} \mathrm{C}$ or $350{ }^{\circ} \mathrm{C}$, in an independent high vacuum chamber (pressure during annealing better than $1 \times 10^{-5}$ Torr). Selected samples were also annealed at $425^{\circ} \mathrm{C}$ for $1 \mathrm{~h}$. The ramp-up rate was $20^{\circ} \mathrm{C} / \mathrm{min}$ and the films were freely cooled to room temperature in high vacuum before removal.

Magnetic hysteresis cycles have been obtained for all the samples using vibrating sample magnetometry. The magnetic field was applied either in the plane or perpendicular to the plane of the samples. Fig. 1 shows a representative set of hysteresis cycles: the ones of the $t_{C o F e B}=1.4 \mathrm{~nm}$ samples series, after annealing at $250{ }^{\circ} \mathrm{C}$. It is clear that the magnetic anisotropy evolves upon change of the Ta layer thickness: low thickness results in IPA, as the easy magnetization axis is in the plane of the sample and large thickness in PMA (the easy magnetization axis is perpendicular to the plane of the sample). The anisotropy field $H_{k}$, defined as the hard-axis saturation field and measured at the intersection of the inplane and perpendicular to the plane loops ${ }^{13}$ increases from $-5.6 \mathrm{kOe}$ for $t_{T a}=0.3 \mathrm{~nm}$ (minus sign indicates IPA, whilst positive indicates PMA) to $-0.9 \mathrm{kOe}$ for $t_{T a}=0.6 \mathrm{~nm}$ and $3.4 \mathrm{kOe}$ for $t_{T a}=1 \mathrm{~nm}$.

Similar behavior is observed for $t_{C O F e B}=1.2 \mathrm{~nm}$ samples: low $t_{T a}$ results in IPA and large $t_{T a}(\geq 0.6$ in this case) in PMA. In contrast, the $t_{\mathrm{COFeB}}=0.9 \mathrm{~nm}$ samples only exhibit PMA for $t_{T a}=0.3 \mathrm{~nm}$. Finally, the $t_{C o F e B}=1.8 \mathrm{~nm}$ samples have always IPA for any $t_{T a}$ and any annealing temperature. All the IPA samples have coercive field, $H_{c}$, less than $10 \mathrm{Oe}$ and the PMA samples have $H_{c}$ between $10 \mathrm{Oe}$ and $30 \mathrm{Oe}$, with the value increasing as the annealing temperature increases.

For further characterizing our stacks, we determine their magnetization and dead magnetic layer thickness. In order to calculate these values, the magnetic moment per surface area versus $t_{C O F e B}$ has been plotted for every $t_{T a}$ and annealing temperature (see Fig. 2(a)). Linear fittings to the data yield the $M_{S}$ from the slope and the dead-layer thickness from the $\mathrm{y}$-axis intercept.

$M_{S}$ values do not change substantially as $t_{T a}$ variate. The average $M_{S}$ value is $1279 \pm 71 \mathrm{emu} / \mathrm{cm}^{3}$ as-deposited and $1292 \pm 62 \mathrm{emu} / \mathrm{cm}^{3}$ after annealing at $250^{\circ} \mathrm{C}$. However, there is a small decrease after annealing at $350^{\circ} \mathrm{C}$, as $M_{S}$ is $1201 \pm 62 \mathrm{emu} / \mathrm{cm}^{3}$, probably due to the increased interlayer diffusion. This trend is verified by selected samples annealed at $425^{\circ} \mathrm{C}$ : their magnetization decreased down to $370 \pm 33 \mathrm{emu} / \mathrm{cm}^{3}$ and $830 \pm 38 \mathrm{emu} / \mathrm{cm}^{3}$ for $\mathrm{CoFeB}$ thickness equal to $1.2 \mathrm{~nm}$ and $1.4 \mathrm{~nm}$, respectively.

Fig. 3 shows the dead layer thickness values as a function of $t_{T a}$ and annealing temperature. The overall trend

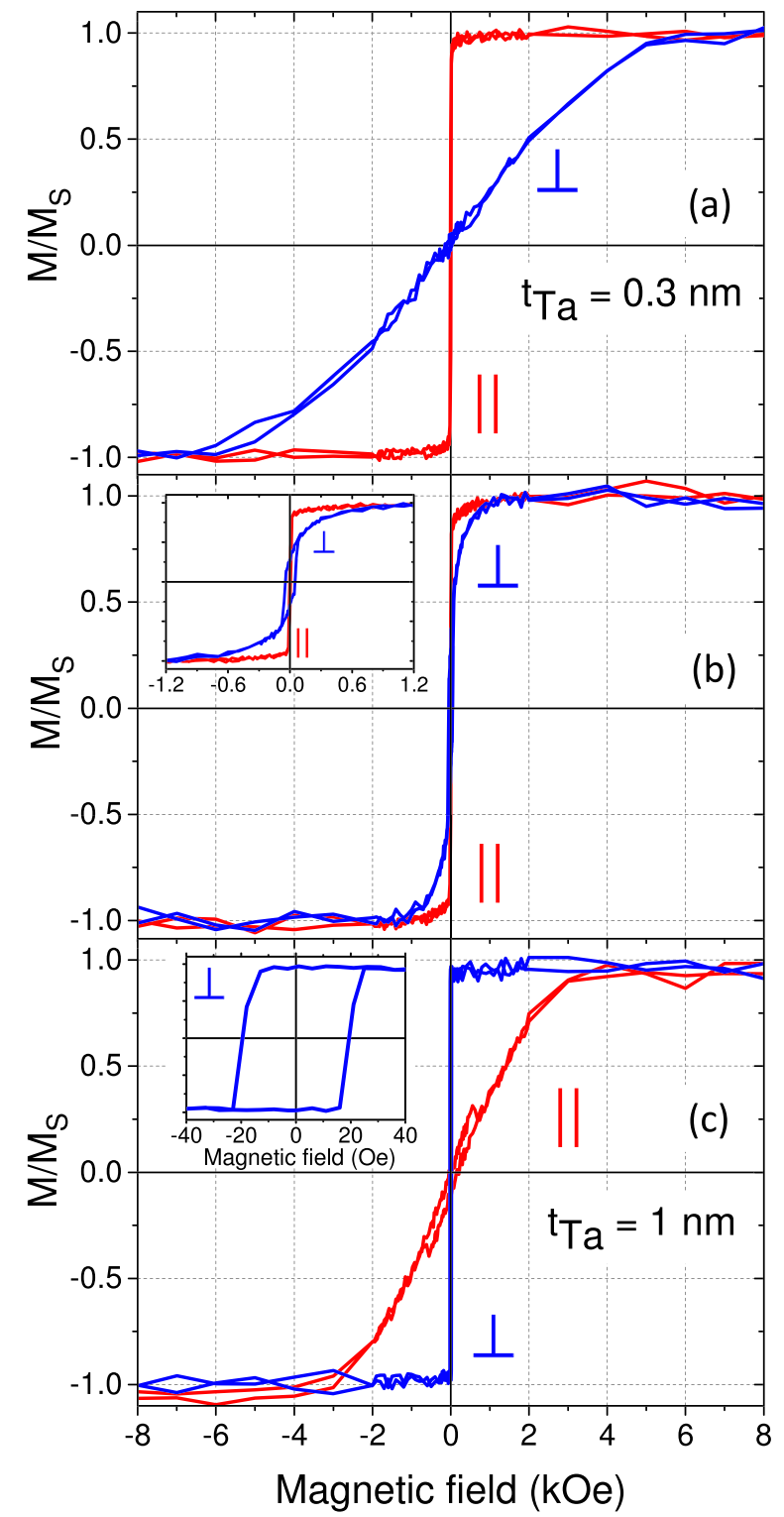

FIG. 1. Representative M-H loops of $t_{\mathrm{CoFeB}}=1.4 \mathrm{~nm}$ stacks annealed at $250{ }^{\circ} \mathrm{C}$ : (a) $t_{T a}=0.3 \mathrm{~nm}$, (b) $t_{T a}=0.6 \mathrm{~nm}$, and (c) $t_{T a}=1 \mathrm{~nm}$. The insets in (b) and (c) show low field regions for clarity.

observed is that the dead-layer thickness is increasing as $t_{T a}$ increases. Also, there is an increase of the dead-layer thickness after annealing, while it remains rather unchanged when increasing the annealing temperature from $250^{\circ} \mathrm{C}$ to $350^{\circ} \mathrm{C}$.

Previous reports show that the dead-layer thickness values depend strongly on the under-layer: $\mathrm{CoFeB} / \mathrm{MgO}$ stacks deposited on $\mathrm{Hf}$ or on $\mathrm{W}$ and $\mathrm{W} / \mathrm{Hf}$ under-layers have almost zero dead-layer thickness. ${ }^{10,14}$ However, $\mathrm{CoFeB} / \mathrm{MgO}$ stacks have dead-layer thickness ranging from 0.5 to $1.1 \mathrm{~nm}$, when deposited on Ta under-layers. ${ }^{12,15}$ In our stacks, we find a small dead-layer thickness $(0.07 \mathrm{~nm}$ before annealing up to almost $0.3 \mathrm{~nm}$ after annealing at $350^{\circ} \mathrm{C}$ ) at the $\mathrm{CoFeB} / \mathrm{MgO}$ stacks deposited directly on $\mathrm{W}$, contrary to previous reports. However, in accordance with the previous studies, it appears that Ta increases the dead-layer thickness reaching a maximum value for $t_{T a}=1 \mathrm{~nm}: 0.25 \mathrm{~nm}$ before annealing and $0.65 \mathrm{~nm}$ after annealing at $350^{\circ} \mathrm{C}$.

For investigating the effect of $t_{T a}$ and annealing temperature on the magnetic anisotropy of the $\mathrm{W} / \mathrm{Ta} / \mathrm{CoFeB} / \mathrm{MgO}$ 

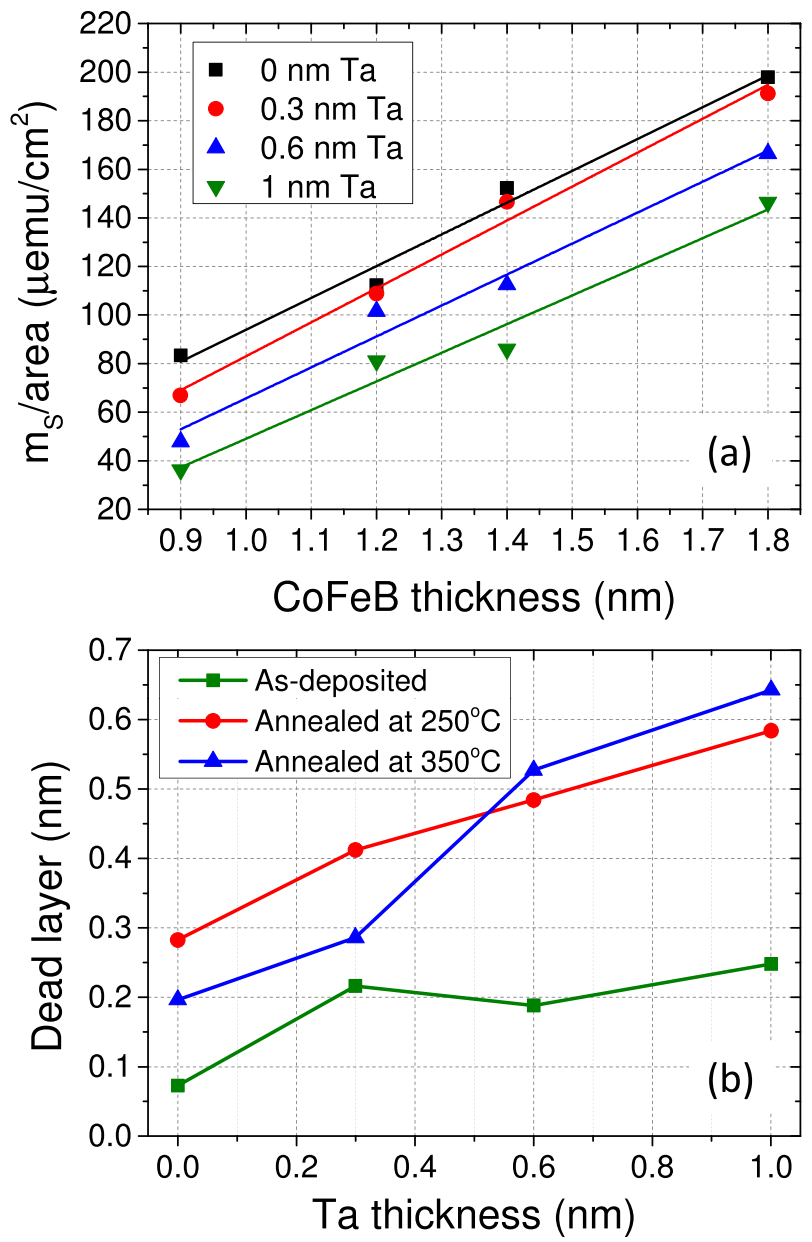

FIG. 2. (a) Representative magnetic moment per surface area versus $t_{C o F e B}$ plot for the samples annealed at $250{ }^{\circ} \mathrm{C}$. Solid lines represent linear fits to the data. (b) Dead-layer thickness variation as a function of $t_{T a}$ and annealing temperature.

system, we determine for each sample its effective magnetic anisotropy energy, $K_{\text {eff }}$ (separated in a volume contribution, $K_{v}$ and a contribution from the interfaces, $K_{s}$ ), which approximately obeys the relation: ${ }^{13}$

$$
K_{e f f}=K_{v}+\frac{K_{s}}{t_{C o F e B}} .
$$

As it has been shown, ${ }^{14} K_{s}$ solely originates from the $\mathrm{CoFeB} / \mathrm{MgO}$ interface, thus, Eq. (1) takes into account only one interface. Finally, $K_{\text {eff }}$ is negative (positive) for IPA (PMA) and can be determined experimentally ${ }^{13}$ using the formula $K_{e} f f=H_{k} M_{s} / 2$.

Fig. 3 shows the variation of $K_{\text {eff }}$ as a function of $t_{C o F e B}$ and $t_{T a}$, before and after annealing. Samples with $t_{T a}=0$ have always IPA for any $t_{\mathrm{CoF} e B}$, before and after annealing. Additionally, samples with $t_{\mathrm{COFeB}}=1.8 \mathrm{~nm}$ have also IPA, for any $\mathrm{Ta}$ thickness, before and after annealing, indicating that this CoFeB thickness is above the threshold where volume anisotropy prevails over the $\mathrm{CoFeB} / \mathrm{MgO}$ interface anisotropy. Samples with $t_{T a}=0.3 \mathrm{~nm}$ have IPA in all cases, except for $t_{C o F e B}=0.9 \mathrm{~nm}$ : in this case, the sample develops PMA after annealing at $250{ }^{\circ} \mathrm{C}$, which is sustained even after annealing at $350^{\circ} \mathrm{C}$. Finally, samples with $t_{T a}=0.6 \mathrm{~nm}$ or $1 \mathrm{~nm}$ have PMA even as-deposited (for $t_{\mathrm{CoF} e B}=1.2 \mathrm{~nm}$ ) and after

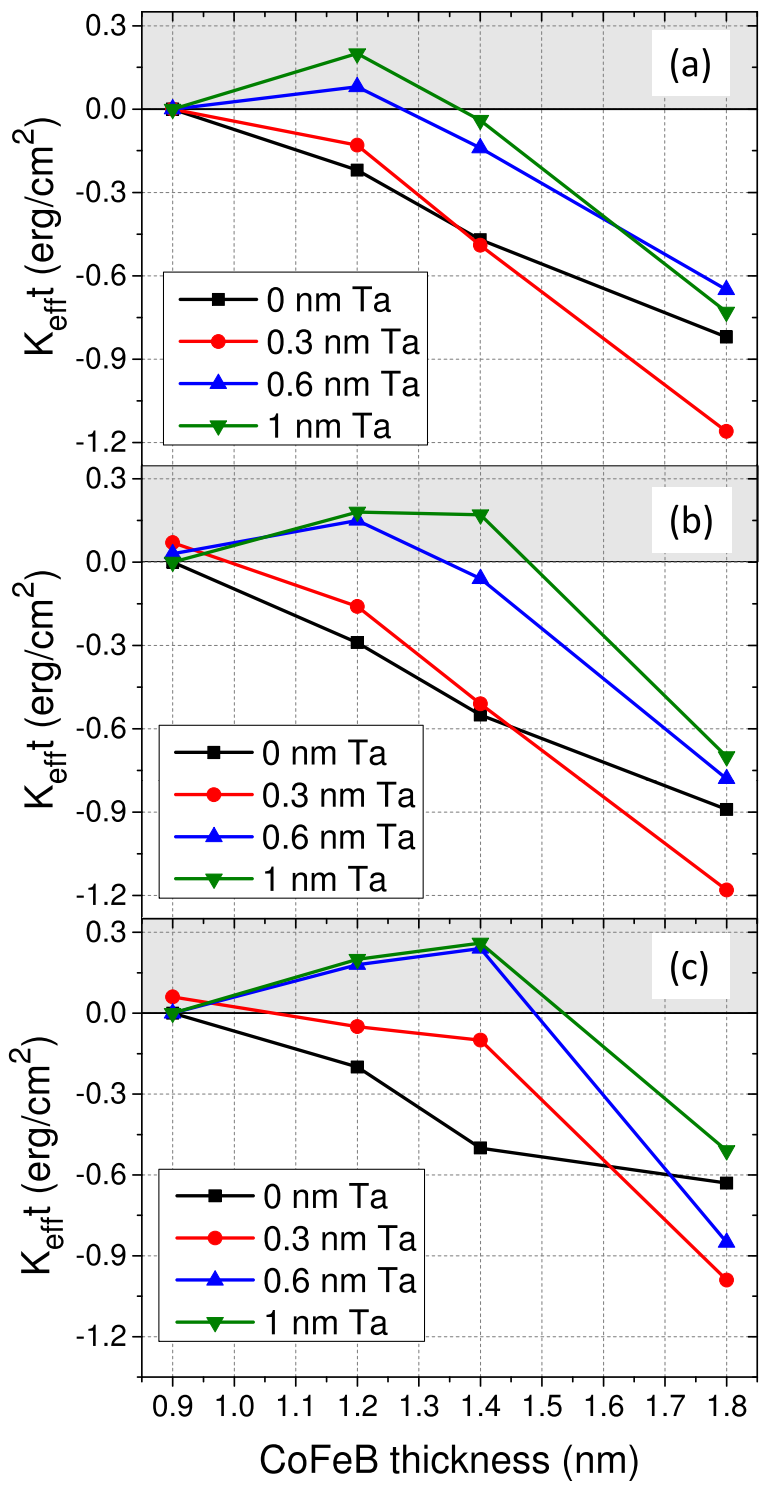

FIG. 3. Effective anisotropy energy $K_{\text {eff }}$ as a function of $t_{\mathrm{CoFeB}}, t_{\mathrm{Ta}}$, and annealing temperature. As-deposited (a) annealed at $250{ }^{\circ} \mathrm{C}$ (b) and annealed at $350^{\circ} \mathrm{C}$ (c) samples. Shaded regions correspond to samples having PMA.

annealing for $t_{C o F e B}=1.2 \mathrm{~nm}$ or $1.4 \mathrm{~nm}$, while their PMA energy increases as the annealing temperature increases.

The maximum PMA anisotropy energy, $3.6 \times 10^{6} \mathrm{erg} / \mathrm{cm}^{3}$, is obtained for $t_{T a}=1 \mathrm{~nm}$ and $t_{C o F e B}=1.4 \mathrm{~nm}$. This value is slightly smaller than the maximum anisotropy energy measured at $\mathrm{W} / \mathrm{Hf} / \mathrm{CoFeB} / \mathrm{MgO}$ samples $\left(4 \times 10^{6} \mathrm{erg} / \mathrm{cm}^{3}\right.$ observed for $\left.t_{C o F e B}=1.1 \mathrm{~nm}\right)^{10}$ and considerably smaller than the maximum value measured at a $\mathrm{Hf} / \mathrm{CoFeB} / \mathrm{MgO}$ samples $\left(6 \times 10^{6} \mathrm{erg} / \mathrm{cm}^{3}\right.$ observed for $\left.t_{C o F e B}=1.1 \mathrm{~nm}\right) .{ }^{14}$ However, it should be noted that $\mathrm{Hf}$ is inadequate material for SOT-MRAM applications due to its low spin-orbit coupling. Finally, the well-studied $\mathrm{Ta} / \mathrm{CoFeB} / \mathrm{MgO}$ system is reported to have values between $1.8 \times 10^{6} \mathrm{erg} / \mathrm{cm}^{3}$ and $4 \times 10^{6} \mathrm{erg} / \mathrm{cm}^{3}$, both for $t_{\mathrm{CoFeB}}=0.9 \mathrm{~nm} .^{12,14}$ The obtained maximum anisotropy energy value in addition to the fact that our $\mathrm{W} / \mathrm{Ta} / \mathrm{CoFeB} / \mathrm{MgO}$ stacks retain their high spin-orbit coupling $\beta-\mathrm{W}$ phase ${ }^{7}$ even after annealing at $350{ }^{\circ} \mathrm{C}$ for $1 \mathrm{~h},{ }^{16}$ make this system highly promising for SOT-MRAM applications. It is worth mentioning that higher annealing temperature does not improve the PMA anisotropy energy: 
samples annealed at $425^{\circ} \mathrm{C}$ have almost zero anisotropy. Besides, $\mathrm{CoFeB} / \mathrm{MgO}$ PMA stacks have been reported to have thermal endurance up to $425^{\circ} \mathrm{C}$ only when deposited on Mo underlayers. ${ }^{17}$

Our data show that the overall effect of post-deposition annealing is to enhance PMA, up to $350^{\circ} \mathrm{C}$, in contrast to previous studies reporting that PMA is destroyed at this temperature. ${ }^{12,18}$ For explaining this disagreement, the exact role of Ta has to be taken into account. As-deposited amorphous $\mathrm{CoFeB}$ layer crystallizes upon annealing in body-centeredcubic (001) structure, coherently to the $\mathrm{MgO}$ (001) layer, giving rise to high interface anisotropy. ${ }^{11}$ In this process, Ta acts as an efficient B getter, enhancing B out-diffusion and allowing for crystallization to occur. Spectroscopic studies have shown that $\mathrm{B}$ piles-up at the $\mathrm{Ta} / \mathrm{CoFeB}$ interface creating a layer of Ta-B mixture. ${ }^{19}$ However, excessive annealing results in Ta diffusion into the $\mathrm{CoFeB}$ layer and up to the $\mathrm{CoFeB} / \mathrm{MgO}$ interface, ${ }^{20}$ which deteriorates the $\mathrm{CoFeB}$ (001) orientation, decreases the $\mathrm{CoFeB} / \mathrm{MgO}$ interface anisotropy, and ultimately destroys PMA.

In our study, all the samples exhibiting PMA show a steady increase of PMA energy after annealing and as the annealing temperature increases from $250^{\circ} \mathrm{C}$ to $350^{\circ} \mathrm{C}$. This could be explained on the basis of the extent of Ta doping: when the Ta inter-layer has an optimum thickness, it provides sufficient $\mathrm{Ta}$ atoms for assisting $\mathrm{B}$ out-diffusion from $\mathrm{CoFeB}$, while keeping Ta diffusion into $\mathrm{CoFeB}$ minimum. That is why the dead layer thickness increases after annealing, while $M_{s}$ remains almost constant. In effect, Ta doping is tailored instead of depositing the $\mathrm{CoFeB} / \mathrm{MgO}$ stack on top of a pure-Ta under-layer, which acts as a constant source of Ta atoms. This also indicates that $\mathrm{W}$ diffusion into $\mathrm{CoFeB}$ is poor, as no PMA deterioration is observed upon annealing. Finally, the fact that stacks with no Ta layer have always IPA indicates that the CoFeB layer remains amorphous after annealing, probably because $\mathrm{W}$ is a poor $\mathrm{B}$ getter. The above point to the fact that there is a favorable window of $\mathrm{CoFeB} /$ Ta ratios which results in PMA of the $\mathrm{W} / \mathrm{Ta} / \mathrm{CoFeB} / \mathrm{MgO}$ stacks. Below this range, the CoFeB layer insufficiently crystallizes; while above, there is an excessive Ta diffusion into $\mathrm{CoFeB}$. In both cases, IPA is observed.

To conclude, we have demonstrated the importance of Ta doping for obtaining $\mathrm{W} / \mathrm{CoFeB} / \mathrm{MgO}$ stacks with PMA. The extent of Ta doping is the primary parameter affecting $\mathrm{CoFeB}$ magnetic anisotropy. There is a window of $\mathrm{Ta} / \mathrm{CoFeB}$ ratio where PMA may emerge. Below this range, Ta is insufficient for inducing $\mathrm{CoFeB}$ crystallization and therefore PMA. Above this range, excessive Ta diffusion into $\mathrm{CoFeB}$ destroys PMA.

Funding from the E.C. through a FP7-ICT project (Grant No. 318144) and CSIC (Ref. i-LINK0783) was acknowledged. Stimulating discussions with Dr. Gilles Gaudin from Spintec, CNRS, France and Professor Pietro Gambardella from ETH, Switzerland are acknowledged.

${ }^{1}$ R. Sbiaa, H. Meng, and S. N. Piramanayagam, Phys. Status Solidi RRL 5, 413 (2011).

${ }^{2}$ S. Sugahara and J. Nitta, Proc. IEEE 98, 2124 (2010).

${ }^{3}$ M. Becherer, G. Csaba, W. Porod, R. Emling, P. Lugli, and D. SchmittLandsiedel, IEEE Trans. Nanotechnol. 7, 316 (2008).

${ }^{4}$ S. Ikeda, K. Miura, H. Yamamoto, K. Mizunuma, H. D. Gan, M. Endo, S. Kanai, J. Hayakawa, F. Matsukura, and H. Ohno, Nat. Mater. 9, 721 (2010).

${ }^{5}$ I. M. Miron, K. Garello, G. Gaudin, P.-J. Zermatten, M. V. Costache, S. Auffret, S. Bandiera, B. Rodmacq, A. Schuhl, and P. Gambardella, Nature 476, 189 (2011).

${ }^{6}$ I. M. Miron, G. Gaudin, S. Auffret, B. Rodmacq, A. Schuhl, S. Pizzini, J. Vogel, and P. Gambardella, Nat. Mater. 9, 230 (2010).

${ }^{7}$ C.-F. Pai, L. Liu, Y. Li, H. W. Tseng, D. C. Ralph, and R. A. Buhramn, Appl. Phys. Lett. 101, 122404 (2012).

${ }^{8}$ Q. Hao and G. Xiao, Phys. Rev. Appl. 3, 034009 (2015).

${ }^{9}$ J. Torrejon, J. Kim, J. Sinha, S. Mitani, M. Hayashi, M. Yamanouchi, and H. Ohno, Nat. Commun. 5, 4655 (2014).

${ }^{10}$ C.-F. Pai, M.-H. Nguyen, C. Belvin, L. V.-Leao, D. C. Ralph, and R. A. Buhrman, Appl. Phys. Lett. 104, 082407 (2014).

${ }^{11}$ S. Yuasa and D. D. Djayaprawira, J. Phys. D: Appl. Phys. 40, R337 (2007).

${ }^{12}$ V. Sokalski, M. T. Moneck, E. Yang, and J.-G. Zhu, Appl. Phys. Lett. 101, 072411 (2012).

${ }^{13}$ M. T. Johnson, P. J. H. Bloemen, F. J. A. den Broeder, and J. J. de Vries, Rep. Prog. Phys. 59, 1409 (1996).

${ }^{14}$ T. Liu, J. W. Cai, and L. Sun, AIP Adv. 2, 032151 (2012).

${ }^{15}$ D. C. Worledge, G. Hu, D. W. Abraham, J. Z. Sun, P. L. Trouilloud, J. Nowak, S. Brown, M. C. Gaidis, E. J. OSullivan, and R. P. Robertazzi, Appl. Phys. Lett. 98, 022501 (2011).

${ }^{16}$ A. Kaidatzis, V. Psycharis, J. M. García-Martín, C. Bran, M. Vázquez, and D. Niarchos, in Symposium M - Materials and Technology for Nonvolatile Memories, edited by P. Dimitrakis, Y. Fujisaki, G. Hu, and E. Tokumitsu (Mater. Res. Soc. Symp. Proc., 2015), Vol. 1729.

${ }^{17}$ T. Liu, Y. Zhang, J. W. Cai, and H. Y. Pan, Sci. Rep. 4, 5895 (2014).

${ }^{18}$ H. Meng, W. H. Lum, R. Sbiaa, S. Y. H. Lua, and H. K. Tan, J. Appl. Phys. 110, 033904 (2011).

${ }^{19}$ T. Zhu, Y. Yang, R. C. Yu, H. Ambaye, V. Lauter, and J. Q. Xiao, Appl. Phys. Lett. 100, 202406 (2012).

${ }^{20}$ S. Ikeda, J. Hayakawa, Y. Ashizawa, Y. M. Lee, K. Miura, H. Hasegawa, M. Tsunoda, F. Matsukura, and H. Ohno, Appl. Phys. Lett. 93, 082508 (2008). 